\title{
Beta-Cyclodextrin: Eugenol Inclusion Complexes: Characterization and Antifungal Capacity
}

\section{Complejos de Inclusión Beta-Ciclodextrina: Eugenol: Caracterización y Capacidad Antifúngica}

\section{Muñoz-Shugulí*, F. J. Rodríguez, A. Guarda, and M. J. Galotto}

VII International Congress of Science, Technology, Entrepreneurship and Innovation (SECTEI 2020)

Corresponding Author:

C. Muñoz-Shugulí

cristina.munoz.s@usach.c

Published: 26 August 2021

Production and Hosting by Knowledge $E$

(c) C. Muñoz-Shugulí et al. This article is distributed under the terms of the Creative Commons Attribution License, which permits unrestricted use and redistribution provided that the original author and source are credited.
Centro de Innovación en Envases y Embalajes - LABEN Chile, Centro de Desarrollo para la Nanociencia y Nanotenología, Departamento de Ciencia y Tecnología de los Alimentos, Facultad Tecnológica, Edificio de Alimentos, Estación Central, Santiago, Chile

\section{Abstract}

Eugenol (EUG) is the principal component of clove essential oil. It has demonstrated excellent antifungal properties against $B$. cinerea, one of the most important fungus in the fresh fruit decay. However, this substance is highly volatile, thermolabile and the direct contact with a food induce undesirable changes in the organoleptic properties. For this reason, the application of EUG represents a big challenger and its encapsulation through inclusion complexes formation with $\beta$-cyclodextrin ( $\beta-C D)$ is presented as a solution. In this way, the aim of this work was to develop, characterize and assess the antifungal capacity of inclusion complexes $\beta$-CD:EUG. For this, co-precipitation was used as method of inclusion complexes synthesis. The quantity of entrapped EUG was determined by gaseous chromatography. The inclusion complexes were characterized by thermogravimetric analysis (TGA) and Fourier transform infrared (FTIR). Moreover, the antifungal activity was probed in a headspace system. Results showed that the EUG entrapped quantity was of $68,5 \mathrm{mg} / \mathrm{g}$ inclusion complexes. Furthermore, the characteristic peaks of EUG did not appear in inclusion complexes TGA thermogram and FTIR spectrum which confirm the effective compound encapsulation. Finally, inclusion complexes inhibited the growing of $A$. cinerea at $53 \%$ and avoided the fungal sporulation. These findings suggest that the $\beta$-CD:EUG inclusion complexes are suitable to use in the storage and transportation of fresh fruits to prevent their deterioration.

Keywords: Botrytis cinerea, Fresh fruits, Headspace.

\section{Resumen}

El eugenol (EUG) es el principal componente del aceite esencial de clavo de olor. Éste ha destacado por su efectivo control de Botrytis cinerea, uno de los hongos más importantes que provoca la pudrición de frutas frescas. Sin embargo, esta sustancia es altamente volátil, termolábil y provoca cambios desagradables en las propiedades organolépticas del alimento si está en contacto directo con el mismo, por lo que su aplicación presenta un gran desafío. Ante esto, se propone su encapsulación a través de la formación de complejos de inclusión con $\beta$-ciclodextrina ( $\beta-C D)$. En este sentido, el objetivo de la presente investigación fue desarrollar, caracterizar y evaluar la capacidad antifúngica de complejos de inclusión $\beta$-CD:EUG. Éstos se sintetizaron mediante el método de co-precipitación, y la cantidad de EUG atrapada en la $\beta$-CD fue cuantificada por cromatografía de gases. Los complejos de inclusión se caracterizaron a través de análisis termogravimétrico (TGA) y espectroscospía infrarroja con transformada de 
Fourier (FTIR). Además, su actividad antifúngica se evaluó en un sistema de espacio de cabeza. Los resultados mostraron que la cantidad de EUG atrapada fue de $68,5 \mathrm{mg} / \mathrm{g}$ de complejo de inclusión. Además, en su termograma TGA y espectro FTIR no se observaron los picos característicos del EUG, confirmando la efectiva encapsulación del compuesto. Finalmente, los complejos de inclusión inhibieron el $53 \%$ del crecimiento de $B$. cinerea y evitaron su esporulación. Estos resultados permiten sugerir el uso de complejos de inclusión $\beta$-CD:EUG en el almacenamiento y transporte de frutas frescas para prevenir su deterioro.

Palabras Clave: Botrytis cinerea, frutas frescas, espacio de cabeza.

\section{Introducción}

En la actualidad, la calidad y seguridad alimentaria son asuntos de preocupación a nivel mundial. Los consumidores prefieren comprar y consumir alimentos frescos con forma, tamaño, color y aroma adecuados, además sin defectos, como cortes, lesiones y sobre todo pudriciones $[1,2]$. Sin embargo, productos frescos con alto valor nutritivo como las frutas, son alimentos muy susceptibles a los daños mencionados, sobre todo a las pudriciones, lo que conlleva a su desperdicio [3, 4].

Según estudios de la Organización de las Naciones Unidas para la Alimentación y la Agricultura (FAO por sus siglas en inglés), entre el 45 y $55 \%$ de la producción mundial de frutas y verduras frescas se desperdicia [5], siendo el grupo de alimentos de mayor pérdida a nivel mundial [6]. La principal razón por la que estos productos son rechazados es su descomposición provocada por la presencia de hongos [7]. Entre ellos destaca Botrytis cinerea, el cual provoca pérdidas económicas de hasta 100 billones de dólares anuales a nivel mundial [8, 9].

Botrytis cinerea es un hongo filamentoso que es responsable de la famosa enfermedad 'podredumbre gris'. Se conoce que el moho afecta a más de 200 especies de cultivos alrededor del mundo, siendo los de mayor importancia económica mundial los de uva, frutilla, y tomate [10]. Se ha descrito a $B$. cinerea como el segundo hongo más importante en cultivos de frutas debido a su facilidad en la reproducción y a que en caso de encontrar condiciones adversas, este hongo forma estructuras de sobrevivencia, denominadas esclerocios [11, 12]. De este modo, el moho puede permanecer inactivo durante largos períodos, y posteriormente activarse al encontrar condiciones propicias para su desarrollo. Por esta razón, los productos frescos aparentemente sanos se deterioran durante su distribución e incluso durante el almacenamiento antes del consumo, lo que provoca las grandes cantidades de desperdicios antes indicadas [13].

Sistemas típicos de conservación de alimentos frescos como la disminución de temperatura, no son efectivas para controlar $B$. cinerea, dado que éste tiene la capacidad 
de vivir incluso a muy bajas temperaturas $\left(-5^{\circ} \mathrm{C}\right)$. Por ello, durante varios años se han usado sistemas que involucran la aplicación de agentes químicos para desinfección antes y durante el almacenamiento del alimento [14]. Dentro de éstos, se ha utilizado la fumigación post-cosecha de los productos con dióxido de azufre o sus derivados. Así también, uno de los sistemas actualmente más empleados es el envasado con almohadillas de metabisulfito de sodio, las cuales al entrar en contacto con la humedad del alimento, generan SO2 como inhibidor del hongo $[15,16]$. Sin embargo, este sistema ha provocado críticas ya que podría causar el blanqueo del producto, y un asunto aún más peligroso es que su uso se ha relacionado con reacciones asmáticas graves en personas sensibles al componente [17].

En consecuencia, es necesario desarrollar agentes que permitan el control de este tipo de microorganismos, alargar la vida útil del producto, que garanticen la calidad pero sobre todo la seguridad de los alimentos y sus consumidores $[2,4,18]$.

En este sentido, los aceites esenciales y sus componentes, mayoritariamente volátiles, son sustancias antifúngicas prometedoras y su reputación de agentes 'naturales' eleva su confiabilidad en los consumidores para ser aplicados en alimentos. En efecto, varios de ellos tienen estatus GRASS, es decir son 'Generalmente Reconocidos Como Seguros' por la Food and Drug Agency - FDA, lo que significa que se pueden usar en productos alimenticios sin mayor aprobación [19]. Dentro de esta amplia gama de sustancias naturales, existen aquellas derivadas de especias que han destacado por su capacidad antifúngica frente a $B$. cinerea y otros hongos que provocan el deterioro de frutas, como es el caso del eugenol.

El eugenol (2-metoxi-4-prop-2-enilfenol) es un fenilpropanoide derivado del guayacol con una cadena alilo sustituida en posición para respecto al grupo hidroxilo (Figura 1). Este es el principal componente del aceite esencial de clavo de olor, se encuentra en una proporción del 75 al 95\% dependiendo de la especie. El eugenol se ha utilizado durante muchos años en aplicaciones odontológicas, médicas e incluso cosméticas [20]. Estudios indican que puede ser utilizado como agente antibacteriano y antifúngico en alimentos. Así, en lo que respecta a su efecto específico frente a $B$. cinerea, Abbaszadeh y col. observaron la inhibición total del hongo a través de ensayos in vitro a una concentración de $450 \mu \mathrm{g} / \mathrm{mL}$ de eugenol [21]. Así mismo, Amiri y col. demostraron que una solución de $2 \mathrm{mg} / \mathrm{mL}$ de eugenol en agar permite el 100\% de inhibición de $B$. cinerea. Luego a través de ensayos in vivo, a la misma concentración, lograron la inhibición del 55 al 60\% del hongo en manzanas [22]. De igual forma, Olea y col. determinaron la concentración inhibitoria máxima de 149 ppm de eugenol para evitar la presencia de $B$. cinerea en tomates [23]. Y Valverde y col. también colocaron 0,5 $\mathrm{mL}$ de EUG en una bolsa que contenía uva, lo que permitió un 95\% de inhibición de pudrición provocada por $B$. cinerea [24]. 
A pesar de presentar muy buenas características antifúngicas, trabajar con este tipo de compuestos conlleva inconvenientes tales como que: (i) su contacto directo con el alimento puede resultar en cambios drásticos en sus propiedades organolépticas (sabor, olor y color) de forma desagradable para el consumidor [25]; (ii) son termolábiles, por lo que no pueden ser aplicados en procesos a altas temperaturas; $y$ (iii) su naturaleza es altamente volátil, lo que impide mantener su efecto antifúngico en el tiempo [26]

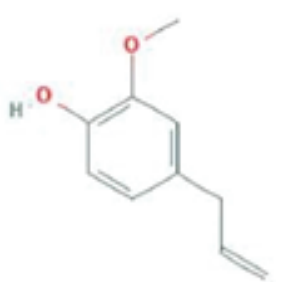

\section{Figure 1}

Estructura química de eugenol (obtenida de plataforma PubChem ${ }^{\circledR}$ ).

Para evitar estos problemas, uno de los mecanismos actualmente más utilizados es la encapsulación del agente volátil a través de la formación de complejos de inclusión con ciclodextrinas [27-30]. Las ciclodextrinas son oligosacáridos cíclicos producto de la hidrólisis enzimática del almidón.

Están formadas por 6, 7 u 8 unidades de D-glucopiranosa ( $\alpha, \beta \circ \gamma$ ciclodextrina) unidas por enlaces $\alpha-1,4$. Tienen una forma cónica hueca y su pared externa es hidrofílica debido a la orientación de los grupos hidroxilo primarios y secundarios (cara primaria y secundaria), lo que las hace solubles en agua. En cambio, los enlaces glucosídicos orientados hacia la cavidad de la molécula hacen que éstas sean considerablemente menos hidrofílicas que la pared (Figura 2), y por tanto, que puedan hospedar a una gran variedad de compuestos hidrofóbicos, como el eugenol, formando así complejos de inclusión [31-33].

Dentro de la familia de ciclodextrinas naturales ( $\alpha, \beta$ y $\gamma$ dextrina), la $\beta-C D$ es la más estudiada y utilizada en la formación de complejos de inclusión debido a su bajo costo, buena disponibilidad y sobre todo el tamaño adecuado de su cavidad (diámetro de $6,0 \AA ̊$ a $6,5 \AA$ Å), lo que permite retener los compuestos volátiles y con ello disminuir la velocidad de liberación.

Además, al formar un complejo de inclusión, el compuesto encapsulado se ve favorecido ya que permite su protección frente a efectos de luz y/o calor, enmascara su olor; mejora su solubilidad al agua y en términos técnicos, mejora su manejabilidad al estar en formato de polvo [31, 34, 35].

Teniendo en cuenta la gran conveniencia en la aplicación de este tipo de agentes en la industria alimentaria, con el fin de preservar alimentos de forma segura, en el 
a.

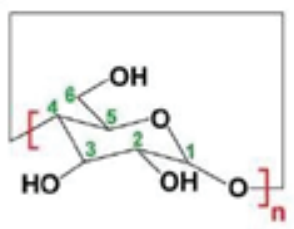

c.

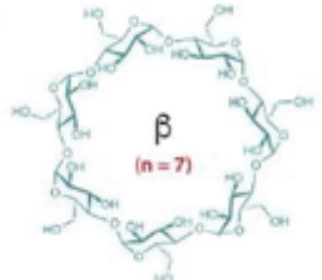

b.
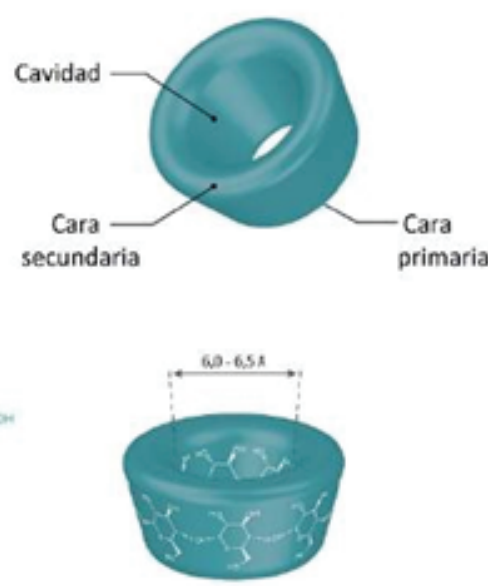

Figure 2

$\beta$-ciclodextrina (a) unidad de D-glucopiranosa, (b) estructura tridimensional y (c) estructura química y dimensiones (obtenida de 31).

presente trabajo se desarrollaron y caracterizaron complejos de inclusión $\beta$-CD:EUG, y se evaluó su capacidad antifúngica frente al hongo $B$. cinerea.

\section{Metodología}

\subsection{Materiales}

Eugenol (EUG) de 164,2 $\mathrm{g} / \mathrm{mL}$ de densidad y $99 \%$ de pureza se obtuvo en Sigma Aldrich Co. ${ }^{\circledR}$ (Santiago, Chile). $\beta$-ciclodextrina ( $\beta-C D$ ) con $98,7 \%$ de pureza se adquirió de CycloLab $^{\circledR}$ (Budapest, Hungría). El acetonitrilo usado fue de grado HPLC y todos los demás reactivos fueron de grado analítico, obtenidos de Merck ${ }^{\circledR}$ (Santiago, Chile). La cepa de $B$. cinerea (B0510) que se utilizó en el presente estudio fue aislada de uva y gentilmente proporcionada por la Laboratorio de Biotecnología Vegetal de la Universidad Andrés Bello, Santiago, Chile.

\subsection{Síntesis de complejos de inclusión}

Para obtener los complejos de inclusión $\beta$-CD:EUG se utilizó el método de co- precipitación. El procedimiento se realizó en base al protocolo de Abarca y col. con ciertas modificaciones [29]. En forma resumida, se diluyó $5 \mathrm{~g}$ de $\beta-C D$ en una disolución de etanol $(30 \% \mathrm{v} / \mathrm{v})$ a $50^{\circ} \mathrm{C}$ con agitación durante $3 \mathrm{hr}$. Luego se añadió EUG en una proporción equimolar $\beta-C D$ :EUG y se agitó durante $30 \mathrm{~min}$. La mezcla se mantuvo en reposo durante $20 \mathrm{hr}$ a $4^{\circ} \mathrm{C}$ para formar los complejos de inclusión por efecto de coprecipitación. Posteriormente el sólido se filtró al vacío junto con una solución de etanol 
(10\% V/V) para eliminar posibles residuos de EUG en las paredes externas de la $\beta-C D$. La fase sólida recuperada se secó por liofilización durante 16 hr, se tamizó en un tamiz \#200 Tyler y se seleccionó la fracción bajo 45 нm, la cual se almacenó en un frasco hermético dentro de un desecador hasta su caracterización.

\subsection{Caracterización de los complejos de inclusión}

Para determinar la cantidad de compuesto activo encapsulado se realizó una extracción del EUG desde $5 \mathrm{mg}$ de complejo de inclusión, con acetonitrilo por 24 hr y agitación a $25^{\circ} \mathrm{C}$. La mezcla se centrifugó y el sobrenadante se analizó mediante un cromatógrafo de gases Perkin Elmer Clarus 500 equipado con una columna capilar ZEBRON ZB-50 (30 $\mathrm{m} \times$ 0,53 mm de diámetro interno con $1 \mu \mathrm{m}$ de espesor de película). La cantidad de EUG se determinó mediante una curva de calibración previamente elaborada con soluciones de 20 a $100 \mathrm{mg}$ EUG/L acetonitrilo $(R=0,997)$.

Además, con el fin de verificar la encapsulación del compuesto en la $\beta-C D$, se realizaron análisis físico químicos a los complejos de inclusión y se compararon con análisis obtenidos de mezclas físicas. Éstas últimas se elaboraron colocando la cantidad de EUG y $\beta-C D$ correspondiente a la síntesis de complejo de inclusión en un mortero y se mezclaron vigorosamente.

Todas las muestras se sometieron a un análisis termogravimétrico. Para esto, 4 a 5 mg de muestra se colocaron en una cápsula de alúmina. Ésta se calentó de 30 a $700^{\circ} \mathrm{C}$ a una velocidad de $10^{\circ} \mathrm{C} / \mathrm{min}$ bajo purga de nitrógeno en un analizador Mettler Toledo TGA/DSC 1 (Schwarzenbach, Suiza).

Adicionalmente, las muestras se analizaron mediante espectroscopía infrarroja con transformada de Fourier (FTIR). Para ello, se prepararon discos de bromuro de potasio $(\mathrm{KBr})$ con muestra al $5 \%(\mathrm{~m} / \mathrm{m})$. Éstos se analizaron en un espectrómetro de transmisión Bruker IFS 66 V (Wismar, Alemania) con resolución de 4 cm-1 y 64 scans. Los espectrogramas se analizaron en un rango de longitud de onda de 4000 a $400 \mathrm{~cm}-1$, rango en el que se verifican los principales grupos funcionales de los componentes.

\subsection{Actividad antifúngica de los complejos de inclusión}

La actividad antifúngica de los complejos de inclusión frente a $B$. cinerea se evaluó mediante un sistema de espacio de cabeza. Para esto, una suspensión de esporas (105 esporas/mL) del moho se colocó sobre un gel de agar papa dextrosa (50 mm diámetro) contenido en el centro de una placa petri de $150 \times 15 \mathrm{~mm}$. Alrededor del gel de agar se colocaron 4, 8 o 12 gramos de complejos de inclusión. Adicionalmente se tuvieron placas sin la presencia de complejos de inclusión y otras con $\beta-C D$, como controles. Cada unidad experimental se ensayó por triplicado. Las placas se sellaron con 
Parafilm ${ }^{\circledR}$ y se almacenaron a $20^{\circ} \mathrm{C}$ por 5 días para permitir el crecimiento del hongo. Los halos radiales de crecimiento se midieron con ayuda del software libre ImageJ (https://imagej.nih.gov/ij/index.html) y se calculó el porcentaje de inhibición mediante la Ecuación 1.

$$
\text { \%de inhibición radical }=\left[\left(x-x_{\#}^{\prime \prime}\right) / x\right] \times 100,
$$

donde $x c$ corresponde al halo promedio en las placas control y $x \mathrm{Cl}$ pertenece al halo de crecimiento de las placas con complejos de inclusión.

\subsection{Análisis estadístico}

Para la evaluación de la actividad antifúngica de los complejos de inclusión se planteó un diseño experimental de 1 factor (cantidad de complejo de inclusión expuesto) con 3 niveles (4, 8 y 12 gramos), cuya variable de respuesta fue el \% de inhibición radial. Los resultados se analizaron mediante un análisis de varianza (ANOVA de una vía) al 95\% de confianza, para detectar posibles diferencias entre tratamientos ( $p<$ 0,05). Posteriormente se aplicó un test de Tukey para obtener las comparaciones entre muestras. Este análisis se llevó a cabo en el software libre Infostat versión 2018 (Córdoba, Argentina).

\section{Resultados y Discusión}

Los complejos de inclusión $\beta-C D$ :EUG se obtuvieron exitosamente mediante el método de co-precipitación. La cantidad de compuesto encapsulada determinada fue de 68,5 \pm 4,74 mg EUG/g complejo de inclusión.

\subsection{Caracterización de los complejos de inclusión}

El análisis termogravimétrico es una técnica relativamente sencilla que permite detectar procesos de degradación y de cambio de estado de los componentes a través de la disminución de la masa de una muestra mientras ésta es calentada a una velocidad controlada (TGA). Además, al graficar la primera derivada de la pérdida de masa respecto a la temperatura (DTG), es posible observar la máxima temperatura a la que ocurre el proceso. En la Figura 3 se muestran ambas curvas tanto para los complejos de inclusión como para la mezcla física. Se observa que la caída de masa en la mezcla física ocurre en 3 etapas. La primera, previo los $100^{\circ} \mathrm{C}$, que corresponde a la evaporación del agua adsorbida por la $\beta-C D$ [36]; la segunda a los $150^{\circ} \mathrm{C}$, correspondiente a la volatilización del EUG [37]; y el tercer proceso, alrededor de los $350^{\circ} \mathrm{C}$, característico de la degradación de la $\beta-C D$ [38]. En cambio, en las curvas TGA y DTG de los complejos 
de inclusión se observan solamente 2 procesos. Aquí, el pico de volatilización del EUG no se distingue, lo cual verifica que el compuesto fue efectivamente encapsulado y protegido por la $\beta-C D$. Además, en la Figura $3 b$ se muestra que el pico de degradación máxima para los complejos de inclusión $\beta$-CD:EUG se encuentra alrededor de los $350^{\circ} \mathrm{C}$, lo cual permite que éstos puedan ser aplicados en procesos que requieran de su exposición a altas temperaturas, sin que el compuesto activo volatilice o se degrade.
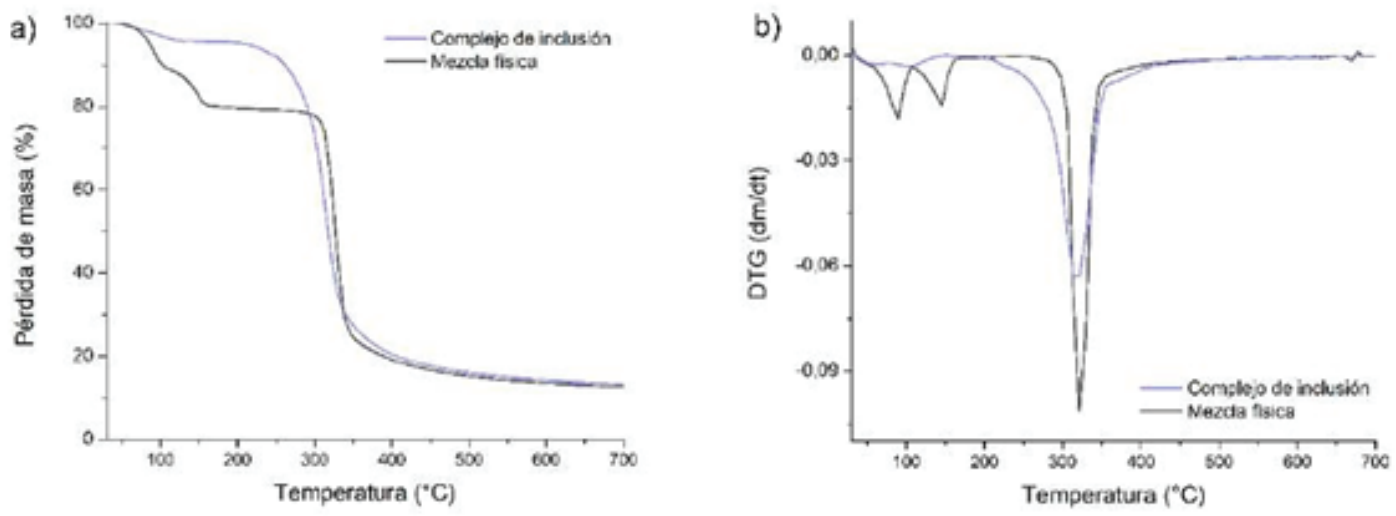

Figure 3

Curvas (a) TGA y (b) DTG de los complejos de inclusión $\beta$-CD:EUG y mezcla física.

Otra forma de comprobar la efectiva encapsulación del EUG en la $\beta$-CD es mediante el análisis FTIR, el cual permite identificar los principales grupos funcionales de las moléculas. Por ejemplo, en la Figura 4 se presenta el espectro perteneciente al EUG, el cual muestra un pequeño hombro a los $3400 \mathrm{~cm}^{-1}$, que corresponde al grupo $-\mathrm{OH}$. En este espectro también se observan un conjunto de bandas entre 2800 y $3000 \mathrm{~cm}^{-1}$, que pertenecen al estiramiento de los enlaces $\mathrm{C}-\mathrm{H}$ del anillo aromático, y otros picos intensos entre 1500 y $1600 \mathrm{~cm}^{-1}$ por la tensión del enlace C-O y C-O-C, enlaces propios de la molécula de EUG (Figura 1) [37, 39]. Por su lado, en el espectro de la $\beta$-CD se observa una banda alta y ancha a los $3400 \mathrm{~cm}^{-1}$ y un conjunto de picos intensos entre los 1000 y $1200 \mathrm{~cm}^{-1}$. Estas señales son características de los grupos hidroxilo $(-\mathrm{OH})$ de la pared externa, $\mathrm{C}=\mathrm{C}$ del anillo y $\mathrm{C}-\mathrm{O}$ del enlace glucosídico en la $\beta-\mathrm{CD}$ (Figura 2 ) $[29,40]$. Así, en el espectro FTIR de la mezcla física se distingue el conjunto de bandas descritas para el EUG entre los 2800 y $3000 \mathrm{~cm}^{-1}$ y también los picos intensos de la $\beta$-CD entre los 1000 y $1200 \mathrm{~cm}^{-1}$. En cambio, en el espectro de los complejos de inclusión no se presentan las señales características del compuesto, sino solamente los picos correspondientes a la $\beta-C D$, lo cual confirma una efectiva encapsulación del EUG al formar los complejos de inclusión. 


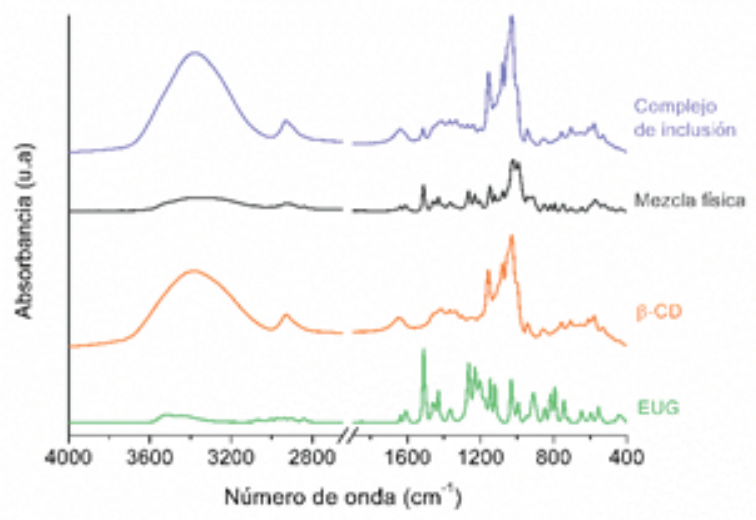

Figure 4

Espectro FTIR de complejos de inclusión ]-CD:EUG, mezcla física, ]-CD pura y EUG puro.

\subsection{Actividad antifúngica de los complejos de inclusión}

El ensayo de actividad antifúngica se realizó mediante un sistema en espacio de cabeza. El volumen de aire libre (espacio de cabeza) dentro de la placa fue de $26,5 \mathrm{~mL}$ y de acuerdo con los resultados obtenidos de la cantidad de EUG encapsulada en los complejos de inclusión, las concentraciones de EUG expuestas fueron de aproximadamente 10, 20 y $30 \mu \mathrm{L} / \mathrm{mL}$ aire para los 4,8 y $12 \mathrm{~g}$ de complejo de inclusión, respectivamente. En la Tabla 1. se resumen los resultados obtenidos, junto con información fotográfica del ensayo. Aquí se confirma que la exposición de $\beta-C D$ no tiene efecto antifúngico, mientras que los complejos de inclusión $\beta$-CD:EUG inhiben el crecimiento de $B$. cinerea entre un 13 a $57 \%$ luego de su exposición por 5 días a $20^{\circ} \mathrm{C}$.

Resultados similares obtuvieron Aguilar-González y col. al inhibir $B$. cinerea a las mismas condiciones con el uso de aceite de clavo de olor, cuyo componente principal fue EUG (75\%). Ellos determinaron que una concentración de 65,6 $\mu \mathrm{L}$ de aceite/mL de aire previene el crecimiento de $B$. cinerea en un 53\% [41]. Dicha concentración duplica a la mayor concentración usada en la presente investigación, y se obtiene un porcentaje de inhibición similar.

Esto probablemente debido a que en nuestro trabajo se utilizó el componente puro, mientras que en el citado se podría asumir que el $25 \%$ de otros componentes en el aceite de clavo de olor no fueron eficientes para inhibir el hongo. Sin embargo, queda en evidencia que la encapsulación del compuesto al formar complejos de inclusión con $\beta$-CD no disminuye su efectividad. Además, es posible postular que, el hecho de encapsular el componente hace que éste se libere paulatinamente, permitiendo así mantener una atmósfera activa y con ello, la inhibición del crecimiento del hongo en el tiempo sin la necesidad de concentraciones altas de compuesto activo. 


\section{Table 1}

Crecimiento e inhibición de $B$. cinerea luego de 5 días a $20^{\circ} \mathrm{C}$, expuesto a distintas muestras mediante un sistema de espacio de cabeza.

\begin{tabular}{|c|c|c|c|}
\hline Muestra & $\begin{array}{l}\text { Crecimiento del } \\
\text { halo } \\
(\mathrm{mm})\end{array}$ & $\begin{array}{l}\text { Inhibición } \\
\text { (\%) }\end{array}$ & $\begin{array}{l}\text { Información } \\
\text { fotográfica }\end{array}$ \\
\hline Control & $46,0 \pm 1,4$ & & \\
\hline $\begin{array}{l}B-C D \\
(8 \mathrm{~g})\end{array}$ & $47,4 \pm 0,6$ & $N^{*}$ & \\
\hline $\begin{array}{lc}\text { Complejo } & \text { de } \\
\text { inclusión } & B- \\
\text { CD:EUG } & \\
(4 \mathrm{~g}) & \end{array}$ & $39,7 \pm 1,5$ & $13,7 \pm 3,3^{a}$ & \\
\hline $\begin{array}{ll}\text { Complejo } & \text { de } \\
\text { inclusión } & B- \\
\text { CD:EUG } & \\
(8 \mathrm{~g}) & \end{array}$ & $29,2 \pm 0,9$ & $36,5 \pm 2,0^{b}$ & \\
\hline $\begin{array}{lr}\text { Complejo } & \text { de } \\
\text { inclusión } & B- \\
\text { CD:EUG } & \\
(12 \mathrm{~g}) & \end{array}$ & $19,7 \pm 1,2$ & $57,2 \pm 2,6^{c}$ & \\
\hline
\end{tabular}

En efecto, en el trabajo realizado por Tsao y col. se observó que a medida que pasa el tiempo, la actividad antifúngica del EUG puro como volátil disminuye. Ellos evidenciaron a que a las 48 hr obtenían un $90 \%$; a las $72 \mathrm{hr}$ un $77 \%$ y finalmente a las $96 \mathrm{hr}$ un 64\% de inhibición de $B$. cinerea. Para dilucidar este hecho, en la presente investigación se decidió tomar el registro de la placa con $12 \mathrm{~g}$ de complejo de inclusión a los 7 días (¡Error! No se encuentra el origen de la referencia. (a) Sin embargo, no es posible hacer una comparación cuantitativa en el \% de inhibición debido a que en la placa control a este tiempo, el hongo creció sobre la totalidad del agar disponible, lo cual se considera un falso control (iError! No se encuentra el origen de la referencia. (b) A pesar de ello, es posible observar que los complejos de inclusión mantienen el efecto antifúngico sobre el crecimiento de $B$. cinerea. Con esto se confirma el hecho 
de que la encapsulación de EUG en complejos de inclusión con $\beta-C D$ ayuda a que el compuesto se libere paulatinamente, permitiendo que se mantenga una atmósfera activa que controle el crecimiento del microorganismo en el tiempo.
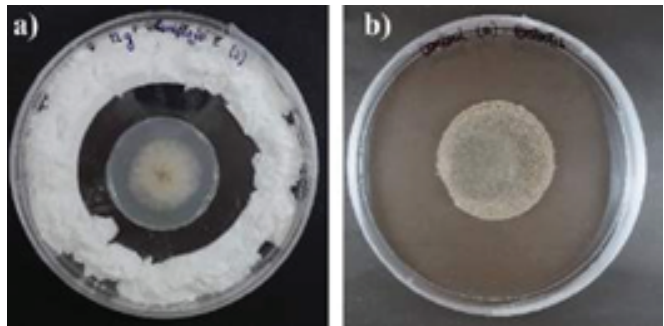

Figure 5

Registro fotográfico del crecimiento de B. cinerea a los 7 días de ensayo. (a) con $12 \mathrm{~g}$ de complejo de inclusión ]-CD:EUG y (b) placa control.

ПPor otra parte, en el registro fotográfico de la Tabla 1. se observa que la presencia de los complejos de inclusión $\beta$-CD:EUG no solamente inhiben el crecimiento, sino que también evitan que el moho produzca esporas (color gris).

Es conocido que, la producción de esporas es el mecanismo por el cual $B$. cinerea se reproduce tan fácil y rápidamente. En efecto, la rapidez del moho para invadir su huésped es uno de los principales problemas en el transporte de fruta fresca [42]. En este sentido, la aplicación de complejos de inclusión $\beta$-CD:EUG en la cadena de suministro de frutas frescas podría contribuir en la disminución de la contaminación de frutas sanas y evitar las altas tasas de pérdida reportadas de estos productos.

\section{Conclusiones}

A través del método de co-precipitación es posible obtener complejos de inclusión $\beta$-CD:EUG con $68,5 \mathrm{mg}$ de EUG/g de complejo de inclusión. Mediante análisis termogravimétrico y espectroscopía infrarroja con transformada de Fourier se confirma la efectiva encapsulación del compuesto en la $\beta$-CD. Por otra parte, mediante ensayos antifúngicos con un sistema de espacio de cabeza se demuestra la efectividad de $\beta$-CD:EUG contra al hongo $B$. cinerea, el control de su crecimiento en el tiempo y la inhibición de su esporulación. De esta manera, se sugiere la aplicación de estos complejos de inclusión en el desarrollo de nuevas tecnologías que contribuyan a mantener la calidad y seguridad, en términos de inocuidad, de frutas frescas durante su distribución y/o almacenamiento. 


\section{Agradecimientos}

Los autores agradecen la Agencia Nacional de Investigación y Desarrollo (ANID-Chile) - Beca Doctorado Nacional No. 21190326 y al Fondo Nacional de Desarrollo Científico y Tecnológico (FONDECYT-Chile) - Proyecto No. 1180686.

\section{Conflicto de Intereses}

Los autores declaran no tener conflicto de intereses.

\section{References}

[1] Sivakumar D, Bautista-Baños S. A review on the use of essential oils for postharvest decay control and maintenance of fruit quality during storage. Crop Prot. 2014;64:2737.

[2] Lloyd K, Mirosa M, Birch J. Active and intelligent packaging. Encycl Food Chem. 2019:January(1):177-82

[3] World Health Organization. Increasing fruit and vegetable consumption to reduce the risk of noncommunicable diseases. World Health Organization;2019.

[4] Han J-W, Ruiz-Garcia L, Qian J-P, Yang X-T. Food packaging: A comprehensive review and future trends. Compr Rev Food Sci Food Saf. 2018;17(4):860- 77.

[5] Food and Agriculture Organization of the United Nations. Food wastage footprint: Impacts on natural resources - Summary report. Food and Agriculture Organization of the United Nations; 2013.

[6] Food and Agriculture Organization of the United Nations. Global food losses and food waste. Gustavsson J, Cederberg C, Sonesson U, Van Otterdijk R, Meybeck A, editors. Rome: FAO; 2011.

[7] Gao H, Wu W, Chen H, Qin Y, Fang X, Jin TZ. Microbial inactivation and quality improvement of tomatoes treated by package film with allyl isothiocyanate vapour. Int J Food Sci Technol. 2018;53(8):1983-91.

[8] Weiberg A, Wang M, Lin F-M et al. Fungal small RNAs suppress plant immunity by hijacking host RNA interference pathways. Science. 2013;342(6154):118-23.

[9] Zhang Z, Qin G, Li B, Tian S. Knocking Out Bcsas1 in botrytis cinerea impacts growth, development, and secretion of extracellular proteins, which decreases virulence. Mol Plant-Microbe Interact. 2014; 27(6):590-600.

[10] Fillinger S, Elad Y, editors. Botrytis - the fungus, the pathogen and its management in agricultural systems. Cham: Springer International Publishing; 2016. 
[11] Brandhoff B, Simon A, Dornieden A, Schumacher J. Regulation of conidiation in Botrytis cinerea involves the light-responsive transcriptional regulators BCLTF3 and BCREG1. Curr Genet. 2017;63(5):931-49.

[12] Elad Y, Vivier M, Fillinger S. Botrytis - the fungus, the pathogen and its management in agricultural systems. Cham: Springer International Publishing; 2016. Botrytis, the good, the bad and the ugly.

[13] Williamson B, Tudzynski B, Tudzynski P, Van Kan JAL. Botrytis cinerea: The cause of grey mould disease. Mol Plant Pathol. 200;8(5):561-80.

[14] Murray K, Wu F, Shi J, Jun Xue S, Warriner K. Challenges in the microbiological food safety of fresh produce: Limitations of post-harvest washing and the need for alternative interventions. Food Qual Saf. 2017;1(4):289-301.

[15] Ahmed S, Roberto S, Domingues A et al. Effects of different sulfur dioxide pads on botrytis mold in 'Italia' table grapes under cold storage. Horticulturae. 2018;4(4):29.

[16] Saito S, Xiao CL. Evaluation of sulfur dioxide-generating pads and modified atmosphere packaging for control of postharvest diseases in blueberries. Acta Hortic. 2017;(1180):123-8.

[17] Ghoshal G. Recent trends in active, smart, and intelligent packaging for food products. Food Packag Preserv. 2018;343-74.

[18] Ribeiro-Santos R, Andrade M, Ramos de Melo N, Sanches-Silva A. Use of essential oils in active food packaging: Recent advances and future trends. Trends Food Sci Technol. 2017;61:132-40.

[19] Hill LE, Gomes C, Taylor TM. Characterization of beta-cyclodextrin inclusion complexes containing essential oils (trans-cinnamaldehyde, eugenol, cinnamon bark, and clove bud extracts) for antimicrobial delivery applications. LWT - Food Sci Technol. 2013;51(1):86-93.

[20] Pavithra B. Eugenol-A Review. Pharm Sci Res. 2014;6(3):153-4.

[21] Abbaszadeh S, Sharifzadeh A, Shokri H, Khosravi AR, Abbaszadeh A. Antifungal efficacy of thymol, carvacrol, eugenol and menthol as alternative agents to control the growth of food-relevant fungi. J Mycol Med. 2014; 24(2):151-156.

[22] Amiri A, Dugas R, Pichot AL, Bompeix G. In vitro and in vitro activity of eugenol oil (Eugenia caryophylata) against four important postharvest apple pathogens. Int J Food Microbiol. 2008;126(1-2):13-9.

[23] Olea A, Bravo A, Martínez R et al. Antifungal activity of Eugenol Derivatives against Botrytis Cinerea. Molecules. 2019;24(7):1239.

[24] Valverde JM, Guillén F, Martínez-Romero D, Castillo S, Serrano M, Valero D. Improvement of table grapes quality and safety by the combination of modified atmosphere packaging (MAP) and Eugenol, Menthol, or Thymol. J Agric Food Chem. 2005;53(19):7458-64. 
[25] Kramer B, Wunderlich J, Muranyi P. Impact of volatile allyl isothiocyanate on fresh produce. Food Packag Shelf Life. 2018;16:220-4.

[26] Higueras L, López-Carballo G, Hernández-Muñoz P, Gavara R, Rollini M. Development of a novel antimicrobial film based on chitosan with LAE (ethyl- N $\alpha$-dodecanoyl-Iarginate) and its application to fresh chicken. Int J Food Microbiol. 2013;165(3):339 45.

[27] Fenyvesi É, Vikmon M, Szente L. Cyclodextrins in food technology and human nutrition: Benefits and limitations. Crit Rev Food Sci Nutr. 2016;56(12):1981-2004.

[28] Szente L, Fenyvesi É, Szente L, Fenyvesi É. Cyclodextrin-Enabled Polymer Composites for Packaging. Molecules. 2018;23(7):1556.

[29] Abarca RL, Rodríguez FJ, Guarda A, Galotto MJ, Bruna JE. Characterization of betacyclodextrin inclusion complexes containing an essential oil component. Food Chem. 2016;196:968-75.

[30] Herrera A, Rodríguez FJ, Bruna JE et al. Antifungal and physicochemical properties of inclusion complexes based on $\beta$ - cyclodextrin and essential oil derivatives. Food Res Int. 2019;121:127-35.

[31] Crini G, Fourmentin S, Fenyvesi É, Torri G, Fourmentin M, Morin-Crini N. Cyclodextrins, from molecules to applications. Environ Chem Lett. 2018;16(4):136175.

[32] Saifull M, Islam Shishir MR, Ferdowsi R, Tanver Rahman MR, Van Vuong Q. Micro and nano encapsulation, retention and controlled release of flavor and aroma compounds: A critical review. Trends Food Sci Technol. 2019;86:230-51.

[33] Tanwar S, Barbey C, Dupont N. Experimental and theoretical studies of the inclusion complex of different linear aliphatic alcohols with cyclodextrins. Carbohydr Polym. 2019;217:26-34.

[34] Kfoury M, Auezova L, Greige-Gergesa H, Fourmentin S. Promising applications of cyclodextrins in food: Improvement of essential oils retention, controlled release and antiradical activity. Carbohydr Polym. 2015;131:264-72.

[35] Lu X, Chen J, Guo Z et al. Using polysaccharides for the enhancement of functionality of foods: A review. Trends Food Sci Technol. 2019;86:311-27.

[36] Yang Z, Xiao Z, Ji H. Solid inclusion complex of terpinen-4-ol/ $\beta$-cyclodextrin: kinetic release, mechanism and its antibacterial activity. Flavour Fragr J. 2015;30(2):179-87.

[37] da Silva CG, Kano FS, dos Santos Rosa D. Thermal stability of the PBAT biofilms with cellulose nanostructures/essential oils for active packaging. J Therm Anal Calorim. 2019;138(4):2375-86.

[38] Han X, Zhang Z, Shen H, Zheng J, Zhang G. Comparison of structures, physicochemical properties and in vitro bioactivity between ferulic acid- $\beta$ cyclodextrin conjugate and the corresponding inclusion complex. Food Res Int. 2019;125:108619. 
[39] Zheng K, Xiao S, Li W et al. Chitosan-acorn starch- eugenol edible film: Physicochemical, barrier, antimicrobial, antioxidant and structural properties. Int J Biol Macromol. 2019; 135:344-352.

[40] Marques CS, Carvalho SG, Bertoli LD et al. $\beta$-Cyclodextrin inclusion complexes with essential oils: Obtention, characterization, antimicrobial activity and potential application for food preservative sachets. Food Res Int. 2019;119:499-509.

[41] Aguilar-González AE, Palou E, López-Malo A. Antifungal activity of essential oils of clove (Syzygium aromaticum) and/or mustard (Brassica nigra) in vapor phase against gray mold (Botrytis cinerea) in strawberries. Innov Food Sci Emerg Technol. 2015;32:181-5.

[42] Petrasch S, Knapp SJ, van Kan JAL, Blanco-Ulate B. Grey mould of strawberry, a devastating disease caused by the ubiquitous necrotrophic fungal pathogen Botrytis cinerea. Mol Plant Pathol. 2019; 20(6):877-92. 